\title{
Distribuição espacial de Mesadenella cuspidata (Lindl.) Garay (Orchidaceae) em uma floresta ribeirinha em Santa Maria, RS, Brasil
}

\author{
Jean Carlos Budke ${ }^{1,2}$, Eduardo Luís Hettwer Giehl ${ }^{1}$, Eduardo Anversa Athayde ${ }^{1}$ e Renato Aquino Záchia ${ }^{1}$
}

Recebido em 10/03/2002. Aceito em 23/06/2003

\begin{abstract}
RESUMO - (Distribuição espacial de Mesadenella cuspidata (Lindl.) Garay (Orchidaceae) em uma floresta ribeirinha em Santa Maria, RS, Brasil). Mesadenella cuspidata é uma orquídea terrícola encontrada no interior de florestas no Sul do Brasil. Apesar de seu valor ecológico e ornamental, pouco se conhece sobre a biologia desta espécie. Os objetivos deste trabalho foram identificar o padrão de distribuição espacial de $M$. cuspidata e verificar se há correlação entre o número de indivíduos jovens e adultos em uma floresta ribeirinha. Para o levantamento, foram amostradas 60 parcelas de $16 \mathrm{~m}^{2}$ cada. Durante agosto e setembro/2001 foram inventariados os indivíduos jovens e adultos das parcelas. Para identificar-se o padrão de distribuição espacial, utilizaram-se o índice de Morisita (MI) e a razão de variância/ média (R). Foram encontrados 463 indivíduos jovens e 178 adultos. Indivíduos jovens e adultos apresentaram distribuição espacial agrupada, sendo que os adultos ( $\mathrm{IM}=2,17 ; \mathrm{R}=4,52)$ estão mais agrupados que os jovens ( $\mathrm{IM}=1,82 ; \mathrm{R}=7,82)$. O coeficiente de correlação linear encontrado $\mathrm{r}=0,61$, demonstrou que a densidade de indivíduos adultos está altamente relacionada à densidade de indivíduos jovens.
\end{abstract}

Palavras-chave: padrão espacial, tamanho populacional, estádios ontogenéticos, parâmetros populacionais, Orchidaceae

\begin{abstract}
Spatial distribution of Mesadenella cuspidata (Lindl.) Garay (Orchidaceae) in a riparian forest, Santa Maria, RS, Brazil). Mesadenella cuspidata (Lindl.) Garay is a terrestrial orchid found inside Southern Brazilian forests. In spite of its ecological and ornamental value, little information is aviable on its biology. The aim of this work was the identification of the spatial distribution patterns of M. cuspidata in a riparian forest. We have sampled 60 quadrats of $16 \mathrm{~m}^{2}$ each. All the young and adult individuals of the quadrats were included. To identify the pattern of spatial distribution, the Morisita Index (MI) and the variance/mean Reason (R) were used. The linear correlation coefficient was used to verify if there is correlation between populations of the juvenils and adults. From 641 individuals sampled, 463 were juvenils and 178 were adults. Juvenils and adult individuals presented gregarious spatial distribution, but the adults (MI $=2,17 ; \mathrm{R}=4,52$ ) were more gregarious than the juvenils ( $\mathrm{IM}=1,82 ; \mathrm{R}=7,82)$. The high relation between the densities of adults and juvenils had been demonstrated by the value of the linear correlation coefficient found, $r=0,61$.
\end{abstract}

Key words: spatial pattern, population size, ontogenetic stages, population parameters, Orchidaceae

\section{Introdução}

Em uma comunidade vegetal, as plantas encontram-se arranjadas conforme as diversas associações intra e interespecíficas existentes ao longo de sua distribuição natural. O tipo de distribuição espacial que uma espécie apresenta é padrão resultante de vários fatores que interagem entre si (Meirelles \& Luiz 1995; Durigan et al. 2000). Fatores abióticos como o tipo de solo, estresse hídrico, altitude, intensidade luminosa e fatores bióticos como polinizadores, dispersores e espécies competidoras são algumas das variáveis encontradas capazes de afetar o padrão de distribuição espacial de uma espécie (Hubbell 1979; Leite 2001; Lundberg \& Ingvarsson 1998). Portanto, as variáveis ambientais dimensionam o padrão espacial, que pode ser agrupado quando os indivíduos estão próximos uns dos outros; aleatório, com indivíduos distribuídos ao acaso; e regular, quando há intervalos regulares entre os indivíduos (Harper 1977; Araújo et al. 2001).

O estudo de populações fornece informações básicas ao nível específico, sobretudo as referentes à mudança no número de indivíduos numa população (Harper 1977). Tremblay \& Ackerman (2001), trabalhando com orquídeas epifíticas do gênero Lepanthes na Costa Rica, situaram tamanho populacional e distribuição espacial dos indivíduos como parâmetros fundamentais na seleção natural, atuando por outro lado, a deriva genética na diferenciação populacional. Da mesma forma, o estudo de populações em diferentes estádios ontogenéticos fornece informações pertinentes ao longo do ciclo de vida da

\footnotetext{
1 Departamento de Biologia, Universidade Federal de Santa Maria, Faixa de Camobi Km 9, CEP 97105-200, Santa Maria, RS, Brasil

2 Autor para correspondência: Rua Andrade Neves, 956, Centro, CEP 96508-020, Cachoeira do Sul, RS, Brasil (jeancbpb@zipmail.com.br)
} 
espécie (Santos 2002). Assim, o conhecimento dos processos de distribuição espacial das espécies é imprescindível para compreender os mecanismos que regem a grande diversidade encontrada na natureza (Bawa 1992).

Segundo Zimmermam \& Aide (1989), o sucesso reprodutivo está correlacionado com o tempo de vida de cada indivíduo, sendo que este é quase totalmente desconhecido no caso de orquídeas (Tremblay \& Ackerman 2001). Esta relação entre números de indivíduos jovens e adultos é fundamental à compreensão dos padrões populacionais de cada espécie (Wells \& Cox 1991).

Orchidaceae é uma das maiores famílias de plantas com flores, estimando-se a existência de 725 gêneros e 19.500 espécies (Dressler 1993). Mesadenella cuspidata (Lindl.) Garay é uma orquídea terrícola ocorrente no interior de florestas do sul do Brasil. Apesar de seu valor ecológico e potencial ornamental, pouco se conhece sobre sua biologia populacional. $\mathrm{O}$ objetivo deste trabalho foi identificar o padrão de distribuição espacial de $M$. cuspidata e observar se há correlação entre abundância de indivíduos jovens e adultos desta espécie em uma floresta ribeirinha de Santa Maria, RS.

\section{Material e métodos}

Caracterização da área de estudo - Santa Maria localiza-se nas coordenadas $53^{\circ} 45^{\prime} \mathrm{W}$ e $2^{\circ} 40^{\prime}$ 'S, Região da Depressão Central, no Estado do Rio Grande do Sul. A área de estudo é um trecho de floresta ripária, situada no campus da Universidade Federal de Santa Maria (UFSM), na divisa com um núcleo habitacional denominado Vila Maringá. Esta floresta margeia o Arroio Passo das Tropas, que é um afluente do Rio VacacaíMirim e pode ser classificada, de acordo com Tabarelli (1992), como Floresta Estacional Decidual Baixomontana.

O clima da região é do tipo Cfa (temperado úmido), de acordo com a classificação climática de Köppen (Moreno 1961). Dados provenientes da Estação Meteorológica de Santa Maria, localizada no mesmo campus, a 138m de altitude, revelam, no período de 1961 a 1990 , temperatura média de $19,6^{\circ} \mathrm{C}$, com médias mensais variando de $24,8^{\circ} \mathrm{C}$ no mês de janeiro a $14,3^{\circ} \mathrm{C}$ no mês de junho; precipitação anual média de $1.686 \mathrm{~mm}$, com chuvas bem distribuídas durante o ano. O diagrama climático foi construído de acordo com Walter (1986), considerando o armazenamento de água no solo de 100mm (Fig. 1). Segundo Lemos et. al. (1973), os solos são pertencentes à Unidade de Mapeamento Santa Maria, sendo classificados como Brunizem Hidromórfico (textura média, relevo suavemente ondulado, substrato siltito-arenito). Essa Unidade de Mapeamento é constituída por solos medianamente profundos, textura superficial arenosa, friáveis e imperfeitamente drenados.

Coleta dos dados - Foram demarcadas 60 parcelas de $4 \times 4 \mathrm{~m}$, totalizando $960 \mathrm{~m}^{2}$ de área amostrada. As parcelas foram arranjadas em seis transecções, paralelas ao curso do rio, apresentando dez parcelas cada. Tendo sido feito previamente em campo, o acompanhamento da fenologia reprodutiva da espécie, foram caracterizados como "adultos" os indivíduos que apresentavam inflorescências durante o período de observação correspondente à fase reprodutiva da espécie. Na categoria "jovens" foram enquadrados aqueles pertencentes aos demais estádios ontogenéticos. $\mathrm{O}$ inventário de jovens e adultos foi feito durante os meses de agosto e setembro/2001.

Análise dos dados - Para identificar-se o padrão de distribuição espacial, utilizou-se o Índice de Morisita (IM) e a Razão Variância/média (R) (Krebs 1989). Os valores de IM e $\mathrm{R}$ menores que 1,0 indicam a inexistência de agrupamento. Valores de IM e R iguais a 1,0 indicam distribuição regular, e os valores maiores que 1,0 indicam agrupamento. A significância estatística foi constatada através do valor $\chi^{2}$ (Qui Quadrado) para um dado número de graus de liberdade (gl), e a nível de significância, desejados. No presente estudo, para gl (n$1=60-1$ ), obteve-se 87,166 para $\alpha=0,01$. Para verificar se há correlação entre as populações de jovens e adultos, usou-se o coeficiente de correlação linear (r) (Triola

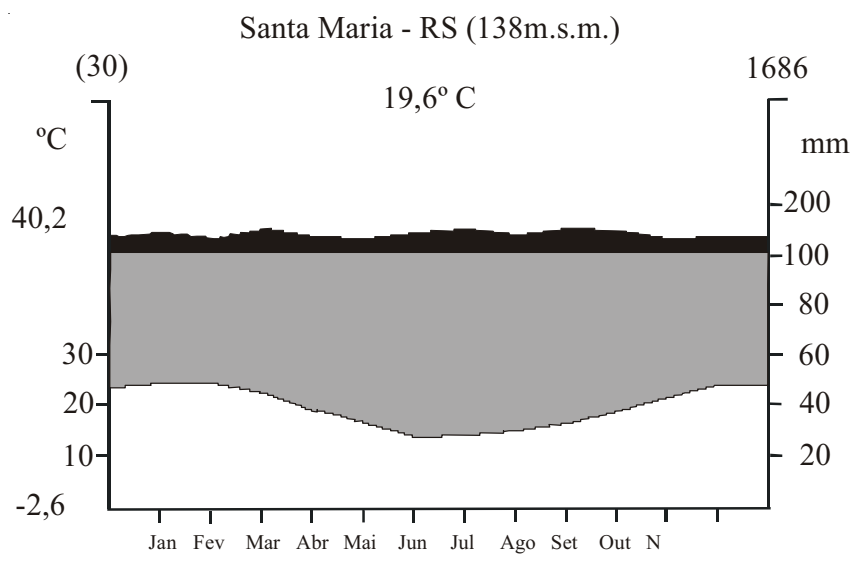

Figura 1. Diagrama climático da região de Santa Maria, RS (534' 'W e 29²0'S, $138 \mathrm{~m}$ de altitude), entre os anos de 1961 e 1990 . 
1999), e a significância do valor obtido verificada através do teste t (Brower \& Zar 1984).

Sendo a Razão (R) calculada por: $R=\frac{S^{2}}{\xi}$

e o índice de Morisita por: $I M=n \frac{\sum x^{2}-N}{N(N-1)}$

considerando que: $n=$ número total de parcelas, $\Sigma x^{2}=$ soma do quadrado do número de indivíduos por parcela; $N=$ número de indivíduos encontrados em todas as parcelas.

A significância dos valores calculados de IM e R é obtida pelo teste de Qui-Quadrado $\left(\chi^{2}\right)$, onde $\chi^{2}$ calculado é:

$$
x^{2}=n\left(\frac{\sum x^{2}}{N}\right)-N
$$

\section{Resultados e discussão}

Distribuição espacial - Foram encontrados 463 indivíduos jovens e 178 adultos, obtendo-se uma população amostrada de 641 indivíduos (Tab. 1). A densidade de jovens e adultos variou de forma considerável entre as parcelas. O número de indivíduos adultos oscilou de zero a 23 por parcela. Esta diferença resultou num $\mathrm{IM}=2,17$ e $\mathrm{R}=4,52$, indicando alta agregação. O número de indivíduos jovens esteve entre zero a 41 por parcela, resultando também num IM = 1,82 e $\mathrm{R}=7,82$, o que também indica padrão de distribuição espacial agrupado aos indivíduos jovens. A significância estatística foi constatada através do valor do Qui-quadrado $\left(\chi^{2}=266,94\right)$ maior que o de tabela, para ambos os índices.

Distribuição agrupada é verificada naturalmente quando, em parcelas semelhantes, o número de indivíduos varia fortemente de uma parcela a outra (Nascimento et al. 2001; Sétamou et al. 2000), a ponto de formarem-se conjuntos de parcelas de acordo com a densidade de indivíduos. Alterando-se o tamanho das parcelas, de $16 \mathrm{~m}^{2}$ para $32 \mathrm{~m}^{2}$, constatou-se leve diminuição nos valores de ambos os índices, mas continuando a agregação dos indivíduos. O IM da população de jovens passou para 1,41 e $\mathrm{R}=7,66$. $\mathrm{Na}$ população de indivíduos adultos, o IM passou para 1,56 e a $\mathrm{R}=4,45$. Novamente, os indivíduos adultos estão mais agrupados do que os jovens.

Analisando-se as populações de indivíduos jovens e adultos, constatou-se que a população de indivíduos jovens está menos agrupada no ambiente. Este fato pode estar associado ao tipo de ambiente onde as plântulas se desenvolveram. De acordo com Hutchings (1986), ambientes heterogêneos levam as plântulas a desenvolverem-se nos sítios mais favoráveis e, assim, populações de estádios ontogenéticos posteriores estariam mais agrupadas. Neste caso, as interações abióticas possuem maior importância nas taxas de mortalidade da população (Hutchings 1986).

A família Orchidaceae possui sementes de tamanho extremamente reduzido, as quais são dispersas pelo vento (Dressler 1993). As sementes caem aleatoriamente sobre o substrato e o estabelecimento de novas plântulas é influenciado por vários fatores, como estresse hídrico, herbivoria e quantidade de luz (Medina 1987). Segundo Migenis \& Ackerman (1993), preferência por substrato, idade e tamanho do substrato podem influenciar a locação de novas plântulas. Ainda, a história da floresta pode ser

Tabela 1. Número de indivíduos jovens e adultos de Mesadenella cuspidata amostrados em cada uma das 60 parcelas em floresta ribeirinha em Santa Maria, RS.

\begin{tabular}{|c|c|c|c|c|c|c|c|c|c|c|c|c|c|c|c|}
\hline Parcelas & 1 & 2 & 3 & 4 & 5 & 6 & 7 & 8 & 9 & 10 & 11 & 12 & 13 & 14 & 15 \\
\hline Jovens & 3 & 2 & 5 & 4 & 4 & 4 & 3 & 8 & 4 & 3 & 8 & 7 & 13 & 6 & 3 \\
\hline Adultos & 2 & 3 & 0 & 0 & 0 & 0 & 0 & 3 & 2 & 4 & 5 & 3 & 8 & 1 & 2 \\
\hline Parcelas & 16 & 17 & 18 & 19 & 20 & 21 & 22 & 23 & 24 & 25 & 26 & 27 & 28 & 29 & 30 \\
\hline Jovens & 12 & 8 & 30 & 15 & 1 & 10 & 5 & 3 & 3 & 5 & 2 & 5 & 1 & 6 & 3 \\
\hline Adultos & 5 & 6 & 7 & 0 & 0 & 7 & 5 & 1 & 0 & 0 & 0 & 4 & 1 & 8 & 1 \\
\hline Parcelas & 31 & 32 & 33 & 34 & 35 & 36 & 37 & 38 & 39 & 40 & 41 & 42 & 43 & 44 & 45 \\
\hline Jovens & 11 & 4 & 6 & 1 & 3 & 1 & 5 & 6 & 8 & 1 & 11 & 3 & 41 & 9 & 3 \\
\hline Adultos & 4 & 1 & 4 & 2 & 0 & 3 & 0 & 1 & 1 & 0 & 0 & 0 & 11 & 4 & 1 \\
\hline Parcelas & 46 & 47 & 48 & 49 & 50 & 51 & 52 & 53 & 54 & 55 & 56 & 57 & 58 & 59 & 60 \\
\hline Jovens & 8 & 6 & 2 & 6 & 10 & 9 & 14 & 12 & 19 & 27 & 4 & 0 & 1 & 24 & 8 \\
\hline Adultos & 4 & 5 & 2 & 3 & 3 & 5 & 4 & 6 & 1 & 5 & 1 & 1 & 5 & 23 & 0 \\
\hline
\end{tabular}


importante na organização espacial de populações e indivíduos, especialmente em se tratando de impactos antrópicos (Tremblay 1997).

Em $M$. cuspidata observou-se a preferência por locais onde o substrato estava menos modificado pelos canais, muito freqüentes em florestas ribeirinhas. Isto condiciona a formação de "ilhas" de substrato em sítios mais elevados que os canais adjacentes. Nessas "ilhas" o estabelecimento de plântulas foi maior, especialmente nas parcelas 59, 46 e 18, sugerindo que os córregos não só afetam o substrato mas, conseqüentemente, a agregação e densidade de novas plântulas.

Correlação entre jovens e adultos - $\mathrm{O}$ coeficiente de correlação linear encontrado entre a densidade de indivíduos jovens e adultos foi de 0,61 (Fig. 2) para indivíduos amostrados em parcelas de $16 \mathrm{~m}^{2}$ e de 0,66 para indivíduos amostrados em parcelas de $32 \mathrm{~m}^{2}$ (Fig. 3). Ambos os resultados foram considerados significativos $(\mathrm{p}<0,01)$. Estes valores indicam que a densidade de indivíduos jovens está altamente associada ao número de indivíduos adultos. Leirana-Alcocer \& Parra-Tabla (1999) encontraram grande correlação entre uma cactácea endêmica, Mammillaria gaumeri na Península de Yucatán no México e a composição do substrato onde as sementes germinavam. Nesse mesmo trabalho, os autores argumentaram que o estabelecimento de plântulas em sítios favoráveis é um dos principais fatores que afetam a distribuição e a densidade daquela espécie.

Correlações positivas entre dois estádios ontogenéticos podem indicar que a variação da densidade de indivíduos adultos ocasiona aumento ou diminuição na densidade de indivíduos jovens, ou seja,

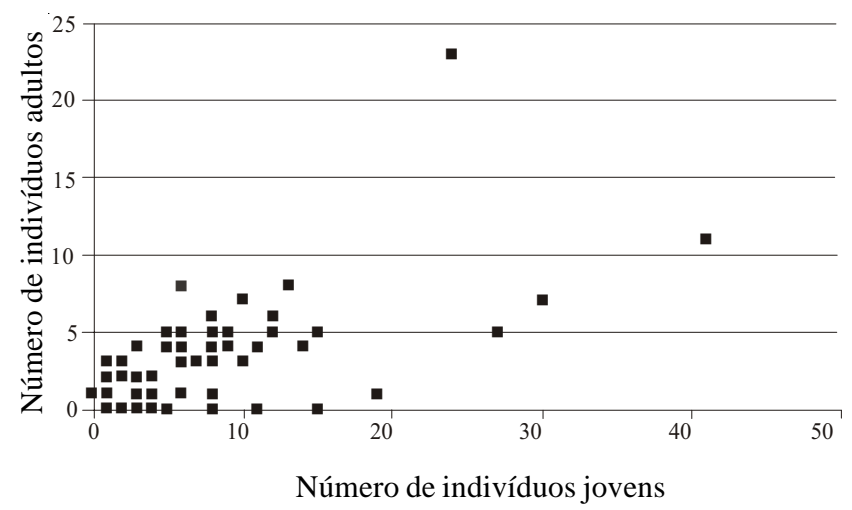

Figura 2. Correlação entre as densidades de indivíduos jovens e adultos de Mesadenella cuspidata em floresta ribeirinha em Santa Maria, RS. Parcelas de $16 \mathrm{~m}^{2}$ resultaram em correlação positiva de 0,61 . Cada ponto do gráfico representa o número de indivíduos adultos e jovens numa determinada parcela.

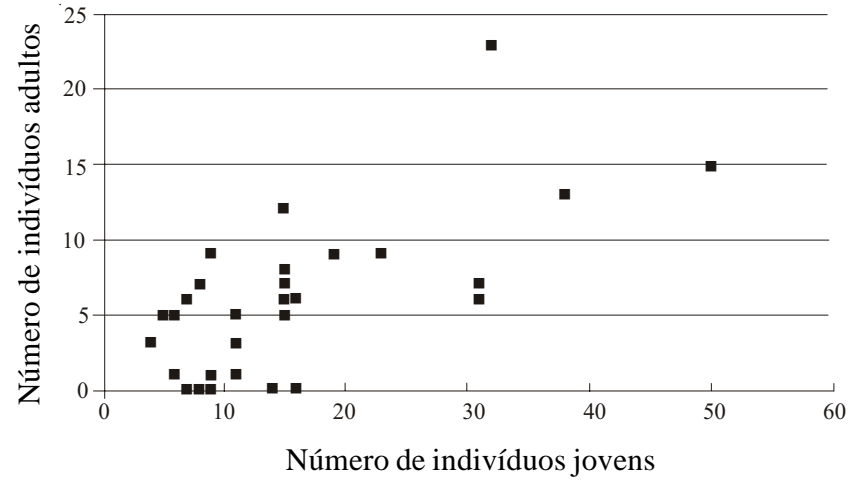

Figura 3. Correlação entre as densidades de indivíduos jovens e adultos de Mesadenella cuspidata em floresta ribeirinha em Santa Maria, RS. Parcelas de $32 \mathrm{~m}^{2}$, resultaram em uma correlação positiva de 0,66. Cada ponto do gráfico representa o número de indivíduos adultos e jovens em determinada parcela.

populações dependentes da densidade, o que pode influenciar no padrão de distribuição espacial da espécie (Hutchings 1986). No entanto, somente com trabalhos com período de execução de longo prazo, esta dinâmica pode ser melhor compreendida.

Estudos relacionados com a distribuição espacial e densidade de orquídeas terrícolas, especialmente com recrutamento e mortalidade, ainda são escassos, tornando difícil a comparação e mesmo o pleno entendimento sobre a biologia destas espécies.

\section{Agradecimentos}

Os autores agradecem ao Fundo de Incentivo à Pesquisa (FIPE, CCNE - UFSM) pelo financiamento do presente estudo; à Profa. Sônia Maria Eisinger, pelas sugestões e leitura crítica do manuscrito; ao Prof. Dari Alves, do Departamento de Zootecnia, pela autorização à realização do trabalho na área; aos revisores anônimos, pelas correções e recomendações.

\section{Referências bibliográficas}

Araujo, M. M.; Osaqui, H. \& Melo, R. S. 2001. Padrão de distribuição espacial de castanheira (Bertholletia excelsa H.B.K.), Barragem do Gelado, Floresta Estacional de Carajás, Pará. Pp. 367-375. In: Anais do II Simpósio Latino-Americano sobre Manejo Florestal. Ed. Universidade Federal de Santa Maria, Santa Maria.

Bawa, K. S. 1992. Mating systems, genetic differentiation, and speciation in tropical rain forest plants. Biotropica 24(2b): 250-255.

Brower, J. E. \& Zar, J.H. 1984. Field \& laboratory methods for general ecology. Wm. C. Brown, Dubuque. 
Dressler, R. L. 1993. Phylogeny and classification of the Orchid family. Dioscorides Press, Portland.

Durigan, G.; Franco, G. A. D. C.; Masahiro, S. \& Baitello, J. B. 2000. Estrutura e diversidade do componente arbóreo da floresta na Estação Ecológica dos Caetetus, Gália, SP. Revista Brasileira de Botânica 23(4): 371-383.

Harper, J. L. 1977. Population Biology of Plants. Academic Press, London.

Hubbell, S. P. 1979. Tree dispersion, abundance, and diversity in a tropical dry forest. Science 203: 1299-1309.

Hutchings, M. J. 1986. The structure of plant population. Pp. 97-136. In: M. J. Crawley (ed.) Plant Ecology. Blackwell Scientific Publ., Oxford.

Krebs, C. J. 1989. Ecological Methodology. Harper Collins, New York.

Leirana-Alcocer, J. \& Parra-Tabla, V. 1999. Factors affecting the distribution, abundance, and seedling survival of Mammillaria gaumeri, an endemic cactus of coastal Yucatán, México. Journal of Arid Environments 41: 421-428.

Leite, E. J. 2001. Spatial distribution patterns of riverine forest taxa in Brasília, Brazil. Forest Ecology and Management 140: $257-264$.

Lemos, R. C.; Azolin, M. D., Abrao, P. R. \& Santos, M. C. L. 1973. Levantamento de reconhecimento dos solos do Estado do Rio Grande do Sul. Boletim Técnico n. 30: Ministério da Agricultura, Departamento Nacional de Pesquisas Agropecuárias, Divisão de Pesquisa Pedológica, Recife.

Lundberg, S. \& Ingvarsson, P. 1998. Population dynamics of resource limited plants and their pollinators. Theorical Population Biology 54: 44-49.

Medina, E. 1987. Aspectos ecofisiológicos de las plantas CAM en los trópicos. Revista de Biología Tropical 35: 55-70.

Meirelles, M. L. \& Luiz, A. J. B. 1995. Padrões espaciais de árvores de um cerrado em Brasília, DF. Revista Brasileira de Botânica 18(2): 185-189.

Migenis, L. E. \& Ackerman, J. D. 1993. Orchid-phorophyte relationship in a forest watershed in Puerto Rico. Journal of Tropical Ecology 9: 231-240.
Moreno, J. A. 1961. Clima do Rio Grande do Sul. Secretaria da Agricultura, Divisão de Terras e Colonização, Porto Alegre.

Nascimento, A. R. T.; Longhi, S. J. \& Brena, D. A. 2001. Estrutura e padrões de distribuição espacial de espécies arbóreas em uma amostra de floresta mista em Nova Prata, RS. Ciência Florestal 11(1): 105-119.

Santos, F. A. M. 2002. Ecologia de populações de plantas região Sudeste do Brasil. Pp. 143-146. In: E. L. Araújo, A. N. Moura, E. V. S. B. Sampaio, L. M. S. Gestinari, J. M. T. Carneiro (orgs.). Biodiversidade, conservação e uso sustentável da flora do Brasil. Editora da Universidade Federal Rural de Pernambuco, Recife.

Sétamou, M.; Schulthess, F.; Poehling, H.M. \& Borgemeister, C. 2000. Spatial distribution and sampling plans for Mussidia nigrivenella (Lepidoptera: Pyralidae) on cultivated and wild host plants in Benin. Environmental Entomology 29(6): 1216-1225.

Tabarelli, M. 1992. Flora arbórea da floresta estacional baixomontana no município de Santa Maria, RS, Brasil. Pp. 260-268. In: Anais do II Congresso Nacional sobre Essências Nativas. Instituto de Biociências, Universidade de São Paulo, São Paulo.

Tremblay, R. L. 1997. Distribution patterns of individuals in nine species of Lepanthes (Orchidaceae). Biotropica 29(1): 38-45.

Tremblay, R. \& Ackerman, J. 2001. Gene flow and effective population size in Lepanthes (Orchidaceae): a case for genetic drift. Biological Journal of Linnean Society 72: 47-62.

Triola, M. F. 1999. Introdução à Estatística. Editora LTC, Rio de Janeiro.

Walter, H. 1986. Vegetação e Zonas Climáticas. Editora Pedagógica Universitária, São Paulo.

Wells, T. C. E. \& Cox R. 1991. Demographic and biological studies on Ophrys apifera: some results from a 10 years study. Pp. 47-61. In: T. C. E. Wells, J. H. Willems (eds.). Population Ecology of Terrestrial Orchids. The Hague: Academic Publishing, The Netherlands.

Zimmerman, J. K. \& Aide, T. M. 1989. Patterns of fruit production in a tropical orchid: Pollinator vs. resource limitation. American Journal of Botany 76: 67-73. 